\title{
Gasparyan Method of Total Autologous Reconstruction of the Aortic Valve
}

\author{
Vahe Gasparyan ${ }^{1}$, A/Prof
}

DOI: $10.21470 / 1678-9741-2020-0197$

\begin{abstract}
In this case report, I describe a new technique for total reconstruction of the aortic valve with autologous pericardium. The parameters of the cusps were calculated using very simple formulas after measurement of the aortic root intercommissural distances. Glutaraldehyde-treated pericardium was trimmed along the marked line, leaving $2 \mathrm{~mm}$ of tissue along the fibrous annulus attachment margin for the suture and small wings on both commissural margins to secure the commissural coaptation between right and noncoronary cusps. The annular margin of each pericardial cusp was sutured to the corresponding fibrous annulus with running $4 / 0$
\end{abstract}

polypropylene suture. The commissures of pericardial patch and the commissural coaptation between right and noncoronary cusps were secured with mattress $4 / 0$ polypropylene sutures. The coaptation of the three cusps was checked with negative pressure on the left ventricular vent before closure of the aortotomy. Intraoperative transesophageal echocardiogram revealed a peak pressure gradient of $10 \mathrm{mmHg}$ and trivial aortic regurgitation.

Keywords: Aortic. Valve Insufficiency. Aortic Valve. Glutaral. Polypropylenes. Echocardiography, Transesophageal. Pericardium. Aorta. Sutures. Running.

\begin{tabular}{ll}
\hline Abbreviations, acronyms \& symbols \\
\hline IL & $=$ Intercommissural distance of left coronary cusps \\
I & $=$ Mean intercommissural distance \\
IN & $=$ Intercommissural distance of noncoronary cusps \\
IR & $=$ Intercommissural distance of right coronary cusps
\end{tabular}

\section{INTRODUCTION}

Total autologous reconstruction of the aortic valve is currently gaining more popularity. I describe a new technique of this operation without any templates, which makes this operation cheaper, easier and more reproducible.

\section{TECHNIQUE OF PROCEDURE}

New technique of total autologous aortic valve reconstruction was used in a 39-year-old patient who presented with septic endocarditis and aortic stenosis (peak pressure gradient through

\footnotetext{
${ }^{1}$ Erebouni Medical Center, Yerevan, Armenia.
}

This study was carried out at the Erebouni Medical Center, Yerevan, Armenia. the aortic valve of $80 \mathrm{mmHg}$ ) complicated with small aortic root $(17 \mathrm{~mm})$ and fibrous annulus abscess. He was treated with antibiotics for 6 weeks before surgery. The patient underwent this operation after obtaining written informed consent. An $8 \times 10 \mathrm{~cm}$ piece of pericardium was harvested after the usual median sternotomy. The harvested pericardium was treated with 0,625\% glutaraldehyde solution for 10 minutes. Cardiopulmonary bypass was established with aortic and right atrial cannulation. The heart was arrested in diastole by retrograde cold blood cardioplegia. The arrest was maintained with retrograde cold blood cardioplegia every 20 minutes. A transverse aortotomy was performed $1 \mathrm{~cm}$ distal from the sinotubular level. After resection of the diseased aortic cusps, the sizes of the intercommissural distances of right (IR), left (IL) and noncoronary (IN) cusps were taken with calipers (Figure 1) and compared to a ruler (to the nearest $0,5 \mathrm{~mm})$. The mean intercommissural distance $\left(I_{\text {mean }}\right)$ was calculated as following:

$$
I_{\text {mean }}=(I R+I L+I N) / 3
$$

Correspondence Address:

Vahe Gasparyan

(ID) https://orcid.org/0000-0002-2613-7158

Erebouni Medical Center, 14, Titogradyan St., Yerevan, Armenia Zip code: 0087

E-mail:vahegasparyan@yahoo.com 
The aortic valve parameters were calculated (Table 1) using my formulas, published in $2000^{6}$ :

$$
\begin{aligned}
& L R=1.2 \times I R \\
& L L=1.2 \times I L \\
& L N=1.2 \times I N \\
& H=0.866 \times I_{\text {mean }} \\
& R=0.6 \times I_{\text {mean }} \\
& K=0.266 \times I_{\text {mean }}
\end{aligned}
$$

where $L R$, $L L$ and $L N$ are the lengths of coapting edge of right, left and noncoronary cusps, respectively; $H$ is the cusps height; $R$ is the annular margin line radius and $K$ is the commissural height. The parameters of the new valve were

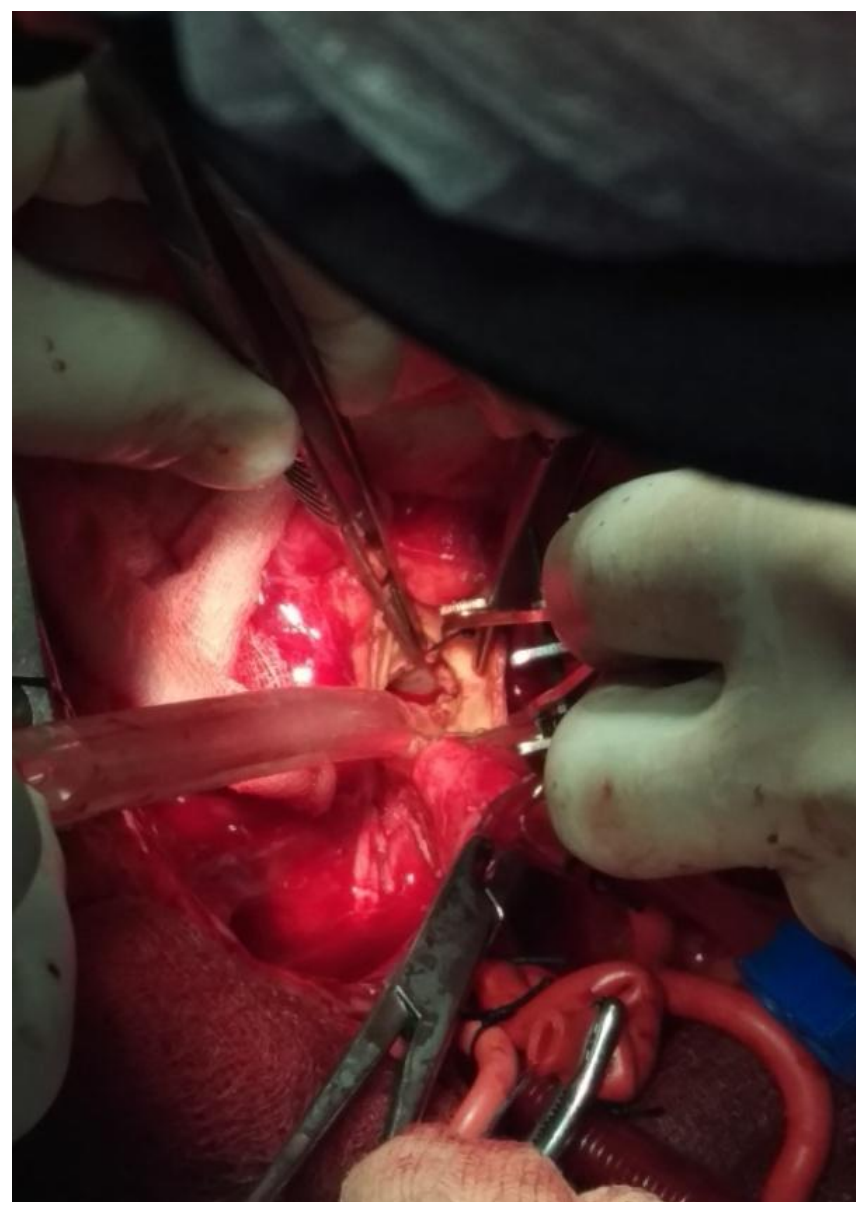

Fig. 1 - Sizing of the intercommissural distances of right, left and noncoronary cusps. marked on the pericardium leaving a distance of about $4 \mathrm{~mm}$ between the cusps (Figure 2) as the pericardial commissures. The glutaraldehyde-treated pericardium was trimmed along the marked line (dashed line in Figure 2) leaving about $2 \mathrm{~mm}$ of tissue along the fibrous annulus attachment margin for the suture and small wings on both commissural margins to secure the commissural coaptation between right and noncoronary cusps. The coapting margins of the pericardial leaflets were trimmed not as a straight line, but slightly as a pyramid-shape line (Figure 2) to raise up the contact point of the new pericardial cusps for better coaptation. The annular margin of each pericardial cusp was sutured to the corresponding fibrous annulus with running 4/0 polypropylene suture (Prolene, Ethicon, Inc., Somerville, NJ) as described in my previous publication ${ }^{[1]}$. The commissures of pericardial patch and the commissural coaptation between right and noncoronary cusps were secured with mattress 4/0 polypropylene sutures as described in my previous publication ${ }^{[1]}$. The smooth (inner) surface of pericardium was placed on the left ventricular side. The coaptation of the three cusps was checked with negative pressure on the left ventricular vent before closure of the aortotomy (Figure 3). Intraoperative transesophageal echocardiography revealed peak pressure gradient of 10 $\mathrm{mmHg}$ and trivial aortic regurgitation. The patient did not take anticoagulants postoperatively. Follow-up transthoracic echocardiography performed 1 and 3 months after surgery revealed a peak pressure gradient of $12 \mathrm{mmHg}$ and grade I aortic regurgitation.

\section{DISCUSSION}

Total reconstruction of aortic valve with glutaraldehydetreated autologous pericardium is currently gaining more popularity. This is a stentless, autologous valve - a very good

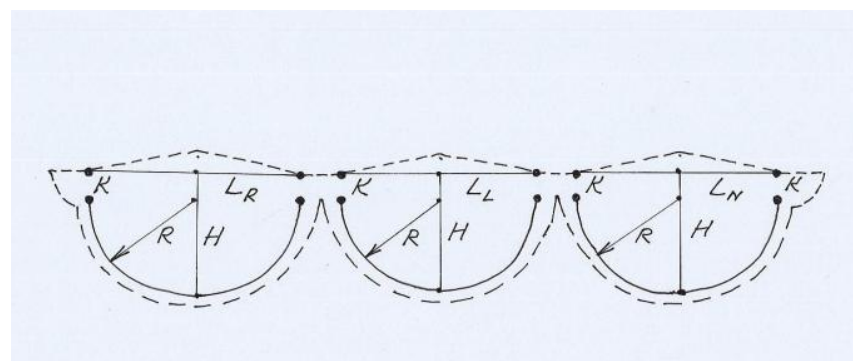

Fig. 2 - Marking of the calculated parameters of the aortic valve in the pericardium ( $L R, L L$ and $L N$ are the lengths of coapting edge of right, left and noncoronary cusps, respectively; $H$ is the cusps height; $R$ is the annular margin line radius and $K$ is the commissural height).

Table 1. Measured and calculated parameters of aortic root and valve cusps.

\begin{tabular}{c|c|c|c|c|c|c|c|c|c}
\hline \multicolumn{1}{c|}{ Measured parameters $(\mathbf{m m})$} & \multicolumn{10}{c}{ Calculated parameters (mm) } \\
\hline $\mathrm{IR}$ & $\mathrm{IL}$ & $\mathrm{IN}$ & $\mathrm{I}_{\text {mean }}$ & $\mathrm{LR}$ & $\mathrm{LL}$ & $\mathrm{LN}$ & $\mathrm{H}$ & $\mathrm{R}$ & $\mathrm{K}$ \\
\hline 14 & 13 & 13 & 13.3 & 17 & 16 & 16 & 12 & 8 & 4 \\
\hline
\end{tabular}




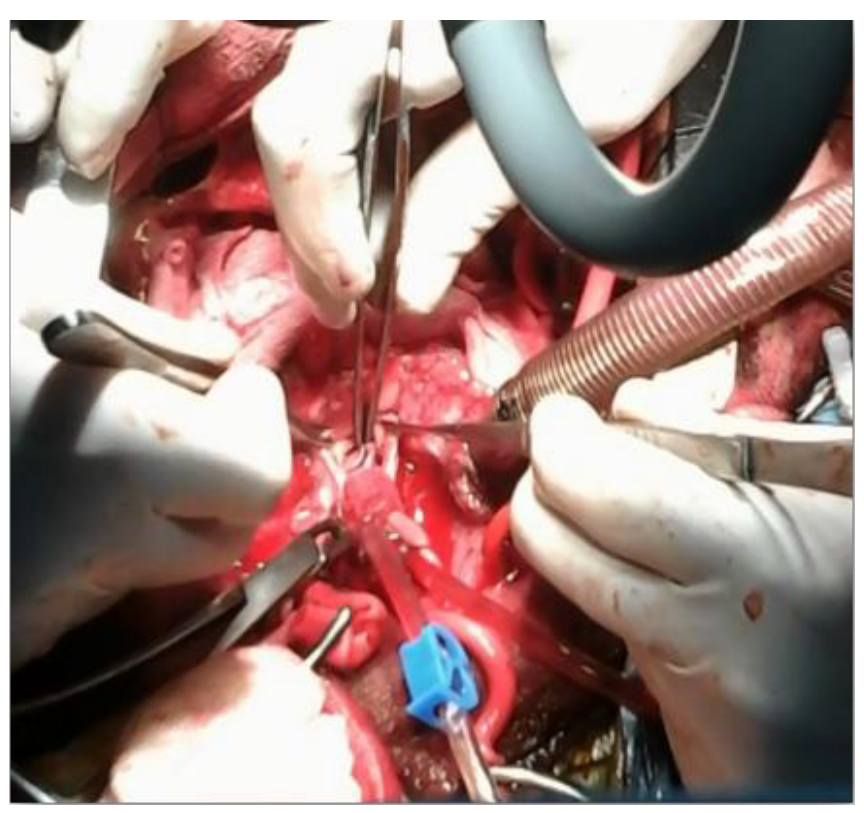

Fig. 3 - Final view of the new pericardial valve sutured to the fibrous annulus of the root. Good coaptation of the pericardial leaflets is seen.

alternative for the prosthetic valves and Ross operation. It was firstly reported by Dr. Duran back in 1995[2]. Special molds of different sizes depending on the aortic root diameter were used to tailor the pericardial patch. Dr. Ozaki has recently reported his experience with this operation using self-developed sizing apparatus and templates for pericardial trimming ${ }^{[3,4]}$. They reported very good results in the medium and long term ${ }^{[3-}$ 5]. All authors still used molds or templates for pericardial patch trimming. The described method is cheaper, easier and more reproducible, since no templates are necessary, and all parameters of the cusps can be calculated using very simple formulas ${ }^{[6]}$. Tailoring of the pericardial valve is not difficult and took only about 10 minutes. The overall cross-clamp time was 76 minutes, which is quite acceptable.

\section{CONCLUSION}

This is an original method of total autologous reconstruction of the aortic valve. It is cheaper and easier than other similar techniques described in the literature, since no molds or templates are necessary and all parameters of the new valve cusps can be calculated using very simple formulas after intraoperative measurement of the root intercommissural distances. I believe that the described technique will make this operation more reproducible.

\section{No financial support. \\ No conflict of interest.}

\section{Author's roles \& responsibilities}

VG Substantial contributions to the conception or design of the work; or the acquisition, analysis, or interpretation of data for the work; drafting the work or revising it critically for important intellectual content; final approval of the version to be published

\section{REFERENCES}

1. Gasparyan VC. Reconstruction of the aortic valve with autologous pericardium: an experimental study. J Thorac Cardiovasc Surg. 1999;117(1):197-8. doi:10.1016/s0022-5223(99)70493-9.

2. Duran CM, Gometza B, Kumar N, Gallo R, Martin-Duran R. Aortic valve replacement with freehand autologous pericardium. JThorac Cardiovasc Surg. 1995;110(2):511-6. doi:10.1016/S0022-5223(95)70248-2.

3. Ozaki S, Kawase I, Yamashita H, Uchida S, NozawaY,Takatoh M, et al. Aortic valve reconstruction using autologous pericardium for ages over 80 years. Asian Cardiovasc Thorac Ann. 2014;22(8):903-8. doi:10.1177/0218492314520748.

4. Ozaki S, Kawase I, Yamashita H, Uchida S, Nozawa Y, Takatoh M, et al. A total of 404 cases of aortic valve reconstruction with glutaraldehyde-treated autologous pericardium. J Thorac Cardiovasc Surg. 2014;147(1):301-6. doi:10.1016/j.jtcvs.2012.11.012.

5. Al Halees Z, Al Shahid M, Al Sanei A, Sallehuddin A, Duran C. Up to 16 years follow-up of aortic valve reconstruction with pericardium: a stentless readily available cheap valve? Eur J Cardiothorac Surg. 2005;28(2):200-5; discussion 205. doi:10.1016/j.ejcts.2005.04.041.

6. Gasparyan VC. Method of determination of aortic valve parameters for its reconstruction with autopericardium: an experimental study. J Thorac Cardiovasc Surg. 2000;119(2):386-7. doi:10.1016/S0022-5223(00)70200-5. 